\title{
Trafficking in cell fate
}

\author{
Sandra Bajjalieh \\ The hyh mutation that underlies a form of congenital hydrocephalus has been traced to the gene encoding $\alpha$ Snap, a \\ membrane trafficking protein. The mutation causes mis-sorting of apical proteins and precocious neurogenesis.
}

The sophisticated neural network that is the mammalian neocortex develops from a precisely timed series of cell divisions. Most cortical neurons arise from the ventricular zone, a region of polarized epithelium adjacent to the lateral cerebral ventricles. During neurogenesis these cells undergo a fixed number of cell divisions that give rise to additional progenitors and to cells that differentiate into neurons $^{1}$. At the beginning of the process, $100 \%$ of cell divisions produce two new progenitors. As development progresses, an increasing percentage of new cells leave the cell cycle and differentiate into neurons. The newly formed neurons migrate up to form the lower, and then the upper, layers of the cortex. As the ratio of new progenitors to neurons dips below $50 \%$ and rapidly proceeds to $0 \%$, there is a burst of neurogenesis that gives rise to the densely packed layers of the upper cortex ${ }^{2}$.

Expanding the progenitor pool through early cell cycles is crucial to generating a sufficient number of neurons to properly form the cortex. Indeed, it has been proposed that a lengthening of this stage during evolution is what gave rise to larger cortices in primates ${ }^{2}$. In congenital hydrocephalus this process goes awry. The reduced cortical thickness and overabundance of deep layer neurons suggests a failure to generate or maintain normal numbers of progenitors. The study of congenital hydrocephalic mouse mutants is providing a window into the genetics of this pathology and the cellular events that regulate cell fate during neurogenesis.

\section{Snap!}

Two new reports, one by Chae et al. ${ }^{3}$ on page 264 and one from Hong et al. ${ }^{4}$, indicate that normal membrane trafficking is required for normal progenitor cell production. Both groups mapped the hyh mutation that gives rise to the hydrocephalus-with-hop gait phenotype in mice. Mice homozygous with respect to this recessive mutation are born with thin

Sandra Bajjalieh is in the Department of Pharmacology, University of Washington, D429 HSB, Box 357280, Seattle, Washington 98195-

7280,USA.e-mail:bajjalie@u.washington.edu cortices that contain an abnormally high percentage of deep-layer (early-born) neurons, leading to hydrocephalus and death within two months of birth. Analyses of the genes in the region traced the mutation to the gene (Napa) that encodes the trafficking protein $\alpha$ Snap. The mutation is a single-base change that leads to the substitution of a highly conserved methionine with isoleucine at posi- tion 105, which is located on the surface of the protein. Chae et al. report that the mutation produces lower levels of Napa mRNA and both groups found lower levels of $\alpha$ Snap protein.

$\alpha$ Snap (soluble N-ethylmaleimide-sensitive factor (NSF) attachment protein) is a ubiquitous membrane trafficking protein present in all eukaryotic cells. Snap proteins bind Snap receptor (SNARE) complexes, the

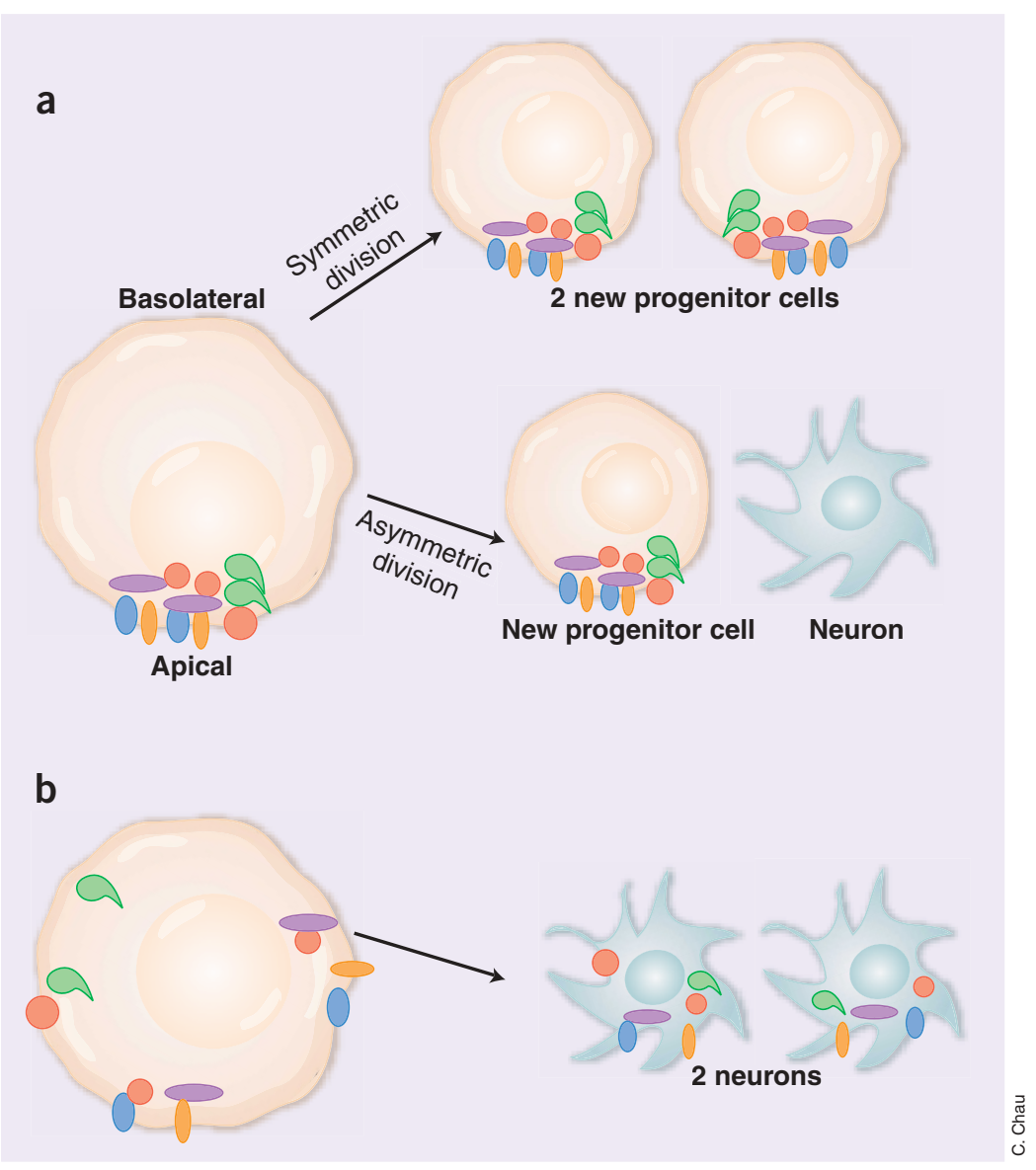

Figure 1 How trafficking might drive cell fate. (a) Normal progenitor cells concentrate soluble factors needed to maintain the progenitor fate at the apical surface, probably anchored to membrane proteins trafficked to the apical surface. Symmetric cell divisions result in two daughter cells that inherit half of the apical domain and half of the basolateral domain. These cells maintain a progenitor fate. Asymmetric divisions produce one daughter that receives the apical domain and one that receives the basolateral domain. This results in one new progenitor (the apical cell) and one cell that exits the cell cycle and becomes a neuron. (b) In hyh mutants, apical proteins are poorly trafficked, resulting in incomplete cell polarization. In this case, cell division doesn't produce cells with sufficient apical factors to maintain a progenitor fate, and both daughters leave the cell cycle to become neurons. 
helical bundles that form between transport vesicles and acceptor membranes and are essential for membrane fusion (reviewed in ref. 5). When it binds the SNARE complex, Snap recruits NSF, an ATPase that catalyzes the disruption of the complex. This frees individual SNARE proteins for another round of complex formation and membrane fusion.

$\alpha$ Snap is an essential protein whose levels must be carefully titrated. Chae et al. report that a null mutation in Napa is lethal in mice, and hypomorphic alleles in Drosophila melanogaster lead to reduced neurotransmission (M. Babcock, G.T. Macleod, J. Leither \& L. Pallanck, personal communication). Overexpression of the protein blocks fusion of yeast vacuoles ${ }^{6}$ and disrupts synapse formation and other aspects of development in D. melanogaster (M. Babcock, G.T. Macleod, J. Leither \& L. Pallanck, personal communication).

The hyh mutation does not affect the gross structure of $\alpha$ Snap or its ability to bind SNARE complexes or to recruit and stimulate NSF-catalyzed disassembly of SNARE complexes. Thus, the mutant phenotype seems to be due to a simple reduction in $\alpha$ Snap levels. This suggests that $\alpha$ Snap has a dose-dependent action during ventricular zone proliferation that is essential to producing neural progenitors.

\section{The importance of being apical}

In early cortical development, two main types of cell division are observed ${ }^{7}$ : symmetric and asymmetric. In symmetric cell divisions both daughters inherit the same complement of membrane and cytoplasm and give rise to two new progenitors. In asymmetric cell divisions one daughter inherits the apical half of the mother cell and remains a progenitor whereas the other daughter inherits the basolateral half and becomes a neuron. The differential partitioning of membrane domains and soluble factors in asymmetric cell divisions is proposed to contribute to differences in cell fate (Fig. 1).

Chae et al. report that in hyh mutants the apical localization of several soluble proteins implicated in cell fate is disrupted and that localization of VAMP7, a vesicular SNARE that participates in apical targeting ${ }^{8}$, is less polarized than in wild-type mice. This suggests that the phenotype could result from a reduction in SNARE-mediated delivery of scaffolding proteins to the apical surface. It also supports a model in which becoming a progenitor is the non-default fate, one that must be actively maintained with sufficient apical signaling machinery. Thus, the decision that must be induced is to prolong proliferation, rather than to initiate differentiation.

Identifying the genes responsible for naturally occurring mutations often leads to sur- prising revelations about cellular processes and to new questions about old players. The seemingly pronounced sensitivity of cortical neurogenesis to levels of $\alpha$ Snap suggests that the shared machinery of membrane trafficking may not function equally throughout the cell. Future studies comparing apical to basolateral sorting in hyh mutant progenitor cells, examining the organization of other polarized tissues and determining whether other Snap isoforms can rescue the phenotype will expand our understanding of snap function. Analyses of other cell fate determinants in hyh mutants will help identify the role these molecules have in the decisions that produce complex cellular patterns. The findings certainly highlight the importance of distinct plasma membrane domains in polarized cells and suggest that they could be orchestrating cell fate determination during neurogenesis.

1. McConnell, S.K. Neuron 15, 761-768 (1995).

2. Caviness, V.S. Jr., Takahashi, T. \& Nowakowski, R.S. Trends Neurosci. 18, 379-383 (1995).

3. Chae, T.H., Kim, S., Marz, K.E., Hanson, P.I. \& Walsh, C.A. Nat. Genet. 36, 264-270 (2004).

4 Hong, H.K., Chakravarti, A. \& Takahashi, J.S. Proc. Natl. Acad. Sci. USA 101, 1748-1753 (2004).

5. Hay, J.C. Exp. Cell Res. 271, 10-21 (2001).

6. Wang, L., Ungermann, C. \& Wickner, W. J. Biol. Chem 275, 22862-22867 (2000).

7. Chenn, A. \& McConnell, S.K. Cell 82, 631-641 (1995).

8. Lafont, F. et al. Proc. Natl. Acad. Sci. USA 96, 3734-3738 (1999)

\title{
Sox3 and sexual dysfunction: it's in the head
}

\author{
Sally A Camper
}

\section{Humans with mutations in SOX3 have panhypopituitarism, but the developmental mechanisms underlying this defect are unknown. Conditional disruption of Sox3 in mice now suggests that anterior pituitary development depends on Sox3 expression in the overlying neural ectoderm, which establishes midline structures and regulates production of inductive BMP and FGF signals.}

\begin{abstract}
Thirteen years ago, Lovell-Badge and colleagues proved that Sry is the mammalian testis-determining gene by showing that Sry was sufficient to convert XX mouse embryos into phenotypic males ${ }^{1}$. Sry is the founding member of a large family of related genes called SOX genes (for SRY-related HMGbox). The SOX DNA-binding domain, called HMG for high mobility group, is the most highly conserved region of these proteins.
\end{abstract}

Sally A. Camper is in the Department of Human Genetics, University of Michigan Medical School, Ann Arbor, Michigan 48109-0638, USA. e-mail: scamper@umich.edu
Do other SOX genes also function in sex determination? Haploinsufficiency with respect to SOX9 causes campomelic dysplasia, a syndrome characterized by skeletal abnormalities and ambiguous genitalia or sex reversal ${ }^{2,3}$. Mice with mutations in $O d s$ and gain of Sox9 function also have sex reversal, identifying the importance of Sox9 gene dosage ${ }^{4}$. Another member of the SOX family, Sox 3 , is closely related to $S r y$, but its role in sex determination is controversial ${ }^{5,6}$. On page 247 Rizzoti et al. report that Sox3, unlike Sry or Sox9, is dispensable for directing the indifferent gonad to develop into an ovary or testis on a mixed genetic background? ${ }^{7}$.
Instead, Sox3 seems to affect gonadal function indirectly through its roles in brain development and hypothalamic induction of anterior pituitary development.

\section{Sox on the brain}

The phenotype of Sox3 mutant mice is variable and complex, with abnormalities throughout the hypothalamic-pituitarygonadal axis (Fig. 1). Severely affected mice have craniofacial abnormalities, defects in the brain midline, profound growth insufficiency, male hypogonadism and lethality, whereas less affected mice are viable and fertile. The expression pattern of Sox3 provided 